G11 THE IMPORTANCE OF USING YOUR HEADSS: INTRODUCTION OF A PROFORMA TO IMPROVE PSYCHOSOCIAL HISTORY TAKING IN THE EMERGENCY DEPARTMENT

KA Murtagh, S Panchal. Paediatric Emergency Medicine, Evelina London/St Thomas' Hospital, Guy's and St Thomas' NHS Foundation Trust, London, UK

10.1136/archdischild-2018-rcpch.11

Aims Psycho-social issues often have a major impact on a Young Person's health, safety and development. Our aim was to improve psychosocial history taking in our busy urban Emergency Department as a step toward overall improvement in the care of Young People within our department and wider community.

Methods We used the internationally recognised HEADSS psycho-social screening tool to audit the emergency attendance record of 60 patients aged 12-17 years, selected at random, attending the Emergency Department of our hospital in November 2016. We assessed if the psychosocial history was recorded for each HEADSS subcategory (Home and environment, Education and employment, Activities, Drugs, Sexuality and Suicide/Depression) and if it was comprehensive, adequate, inadequate or not documented. We also collected information on age, gender, diagnosis, accompaniment and whether they were seen in the Adult or Paediatric Emergency Department. Our initial assessment highlighted a need for urgent and sustained improvement. We therefore introduced an automatically pre-printed HEADSS proforma to the Emergency attendance record for all persons aged 12-17 years. Following this we audited a further 122 sets of notes in July 2017, 62 before a dedicated training sessions on HEADSS and its importance, and 60 after.

Results Prior to the proforma introduction only $3 \%$ of patients had an overall adequate psycho-social history taken. Post introduction this improved to $19 \%$ initially and again to $35 \%$ after dedicated training sessions. Areas better recorded included Home and environment and Educational and employment. Activities, Drugs, Sexuality and Suicide/Depression were more likely to be inadequately assessed. After HEADSS proforma introduction 33\% of practitioners audited used it when assessing Young People. When used the overall adequacy of the psychosocial history obtained improved to $80 \%$.

Conclusion Psychosocial histories are an important part of the assessment of Young People in the Emergency Department. Introducing a pre-printed proforma improved psychosocial history taking in our Department. Better compliance in using the proforma has the potential to drastically improve this overall. Further education and discussion around barriers to its use are required.

\section{G12 ANTIEPILEPTIC DRUG MEDICATION: ARE WE MEETING THE INFORMATION NEEDS OF YOUNG PEOPLE?}

${ }^{1} \mathrm{G}$ Ford, ${ }^{2} \mathrm{~L}$ James, ${ }^{2} \mathrm{~K}$ Spender-Thomas, ${ }^{2} \mathrm{~L}$ Capeling, ${ }^{2} \mathrm{M}$ Barber, ${ }^{2} \mathrm{H}$ Lewis. ${ }^{1}$ School of Medicine, Cardiff University, Cardiff, UK; ${ }^{2}$ Department of Child Health, Aneurin Bevan University Health Board, Newport, UK

\subsection{6/archdischild-2018-rcpch.12}

Aims As part of a youth voice challenge day for Takeover, young people (YP) raised concerns that medicine information leaflets are written for parents and not for the YP. The NICE guideline for the diagnosis and management of the epilepsies recommends that YP be given information about medication and side-effects, in formats suited to their needs. improved understanding may be associated with improved concordance and positive health outcomes. A service evaluation was conducted to establish whether the information needs of YP are being met in relation to anti-epileptic drug (AED) treatment. Methods YP aged 11-16 years within our epilepsy service, who were receiving AED treatment, were surveyed. YP with significant learning disabilities were excluded. A questionnaire was developed to assess their level of understanding, sources of information accessed, information gaps and preferences regarding information formats. YP were asked to evaluate their relevant Medicines for Children leaflet when completing questionnaires in clinic.

Results 23 of 50 eligible YP responded. 6 completed an online survey, 7 completed questionnaires in clinic and 10 returned postal questionnaires. 8 demonstrated good understanding; 8 had gaps in knowledge and 7 poor understanding. 19/23 were able to name their medication. All were able to describe the dosage regime. The majority had vague understanding about how AED treatment works, with some misperceptions. 1/3 had good knowledge of side-effects and managing missed or forgotten doses.

The majority received information from doctors (18/23). Half found this information helpful. 9 accessed no other information, 5 accessed websites, 7 leaflets, 3 sourced information from social media. The majority stated they wished to access information on websites and apps. The main information priorities for YP were how to take medication, how to manage missed medication or illness, ability to drive, length of treatment, and how it would affect the YP at school. Current leaflets were described as 'wordy' and unappealing.

Conclusion Information gaps have been identified amongst the YP surveyed. Current leaflets address some but not all of the information needs identified by YP. YP prefer to access information online. In collaboration with Medicines for Children it is hoped that an area of the website will be developed for YP.

\section{G13 PREVALENCE AND CORRELATES OF LOW MOOD, POOR QUALITY OF LIFE AND HIGH SYMPTOM IMPACT IN ADOLESCENTS ATTENDING A TERTIARY SERVICE FOR CHRONIC FATIGUE SYNDROME/MYALGIC ENCEPHALOMYELITIS}

${ }^{1}$ FK Neale, ${ }^{2}$ TY Segal, ${ }^{3}$ DS Hargreaves. 'Medical School, University College London, London, UK; ${ }^{2}$ Paediatric and Adolescent Medicine, University College London Hospitals NHS Foundation Trust, London, UK; ${ }^{3}$ Paediatric and Adolescent Medicine, University College London Great Ormond Street Institute of Child Health, London, UK

\subsection{6/archdischild-2018-rcpch.13}

Background Chronic Fatigue Syndrome/Myalgic Encephalomyelitis (CFS/ME) is a condition characterised by persistent fatigue that reduces activity and affects everyday life. It is associated with mood disorders, such as depression and anxiety, and a reduction in quality of life.

Aims This project describes the demographic of adolescents with CFS/ME being treated at a specialist service and their mood, quality of life and symptom impact. It assesses whether sex, age, Body Mass Index, household income and illness duration are associated with low mood, poor quality of life and greater symptom impact. It investigates the similarity between the adolescents' and their parents' views of the impact of CFS/ME. 